\title{
Harmonious Wireless Networks: \\ From the Perspective of Interference Management
}

Wei Liu

Wei Liu is with the State Key Laboratory of Integrated Services Networks, Xidian University, China, Email: liuweixd@mail.xidian.edu.cn.

Abstract: In wireless networks, the desired signal of a specific user is typically an undesired interfering signal for untended receivers. However, from the perspective of an entire wireless network, the coexistence of useful signals and interfering signals is unavoidable and often is beneficial in throughput terms. The useful signals and interfering signals are complementary. They are in juxtaposition to each other in the context of a single communication link, while they are in symbiosis within the framework of the networks, which can be described by Chinese traditional culture symbol of "Yin" and "Yang". A wireless network having optimal performance must be a harmonious network, where the interfering and useful signals harmoniously coexist in an optimal balance. Interference management plays the critical role in arriving at this optimal balance.

Keywords: Wireless networks; Duality; Complementarity principle;

"Yin" and "Yang"; Harmonious; Interference management

In $2 \mathrm{G}, 3 \mathrm{G}$ and $4 \mathrm{G}$ systems, orthogonal multiple access schemes have been used for avoiding any multiple access interference. However, in the 5G systems, NonOrthogonal Multiple Access (NOMA) has been advocated, because it has a higher capacity than its orthogonal Multiple Access (OMA) counterparts. 
For 4G, Orthogonal Frequency-Division Multiple Access (OFDMA) is used for downlink transmission and Single-carrier Frequency-Division Multiple Access (SCFDMA) is used for uplink transmission because a single-carrier system has a lower Peak to Average Power Ratio (PAPR) than multiple-carrier systems, hence imposing lower amplifier linearity requirements and thereby facilitating the employment of more power-efficient amplification. Both OFDMA and SC-FDMA can be treated as OMA, where each user is allocated orthogonal sub-carriers, where no overlapping of the subcarriers is allowed, hence avoiding interference among users ${ }^{1}$. However, again for $5 \mathrm{G}$, NOMA has been proposed in order to improve the system's throughput ${ }^{1}$. To elaborate a little further, in Sparse Code Multiple Access (SCMA) ${ }^{2}$, each user is still allocated orthogonal sub-carriers, but the overlapping of sub-carriers is allowed. In this case, interference is encountered among the different users, and yet, SCMA has been shown to improve the system performance ${ }^{2}$.

Another example of improving the throughout in the face of interference is constituted by heterogeneous wireless networks ${ }^{3}$. By deploying tightly packed femtocells and picocells within the coverage area of traditional cellular network over-sailing macrocells, the system's throughput can be improved, despite the cross-tier interference between the small cells and over-sailing macrocells and as well as the inter-cell interference among small cells ${ }^{3}$. 
The key question arising, is whether the interference and the useful signal can or cannot be distinguished or not, not so much whether there exists interference or not. If the interference can indeed be distinguished from the useful signals, then it can be removed by using advanced interference management techniques ${ }^{4,5,6,7,8,9}$. Hence it could be argued that the desired signals and the interference co-exist in symbiosis, which we may also refer to as duality ${ }^{\dagger}$.

\section{Duality of desired and undesired interfering signals}

To elaborate a little further, the desired signal of a specific user is typically an undesired interfering signal for untended receivers. However, from the perspective of an entire wireless network, the coexistence of useful signals and interfering signals is unavoidable and often are beneficial in throughput terms.

To expound further, wireless networks inevitably encounter both useful signals and interfering signals, which are complementary to each other, when viewed from a specific user's perspective. More explicitly, wireless networks cannot be simply viewed as relying only on useful signals or only interfering signals. The useful signals and interfering signals are complementary. They are in juxtaposition to each other in the context of a single communication link, while they are in symbiosis within the framework of the networks.

\footnotetext{
+ This may be deemed reminiscent of the wave vs particle duality of quantum mechanics ${ }^{10}$. 
In traditional Chinese culture, the symbol of "Yin" and "Yang" represents the concept of complementarity. This Chinese traditional symbol of "Yin" and "Yang" has been included in his coat of arms by Bohr to represent his complementarity principle. The philosophy of "Yin" and "Yang" can also represent the complementarity principle of wireless networks as shown in Figure 1, where "Yang" represented by white symbolizes the useful signals and "Yin" in black corresponds to the interfering signals, while the unified circle represents the whole wireless network.

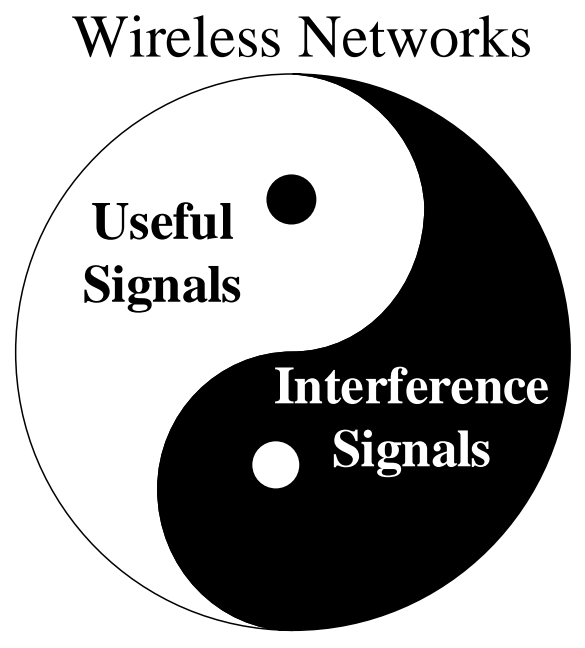

Figure 1 The complementarity principle of wireless networks using "Yin" and "Yang”

Based on the Complementarity principle of wireless networks, a wireless network having optimal performance must be a harmonious network, where the interfering and useful signals harmoniously coexist in an optimal balance. It can be viewed from a pair of perspectives. On the one hand, if there is only interference, the system is useless; on the other hand, if there are only useful signals, the system performance remains 
suboptimal and hence can be improved by tolerating the existence of interference. For example, the system performance can be improved by the evolution from OFDMA to NOMA. In conclusion, interference management plays the critical role in arriving at this optimal balance ${ }^{4,5,6,7,8,9}$.

\section{References}

[1] Wang, Q., Zhang, R., Yang, L. L. and Hanzo, L. Non-orthogonal multiple access: a unified perspective. IEEE Wireless Communications. 25, 2, 10-16 (2018).

[2] Nikopour, H., and Baligh, H., Sparse code multiple access. IEEE International Symposium on Personal Indoor and Mobile Radio Communications, 332-336 (2013).

[3] Yeh, S. P., Talwar, S., Wu, G., Himayat, N. and Johnsson, K. Capacity and coverage enhancement in heterogeneous networks. IEEE Wireless Communications. 18, 3, 32-38 (2011).

[4] B. Soret, Domenico, A. D., Bazzi, S., Mahmood, N. H. and Pedersen, K. I. Interference coordination for $5 \mathrm{G}$ new radio. IEEE Wireless Communications. 25, 3, 131-137 (2018).

[5] Nam, W., Bai, D., Lee, J. and Kang, I. Advanced interference management for 5G cellular networks. IEEE Communications Magazine. 52, 5, 52-60 (2014).

[6] Hossain, E., Rasti, M., Tabassum, H. and Abdelnasser, A. Evolution toward 5G multi-tier cellular wireless networks: an interference management perspective. IEEE Wireless Communications. 21, 3, 118-127 (2014). 
[7] Liu, W., Xue, S., Li, J. and Hanzo, L. Topological interference management for wireless networks, IEEE Access, 6, 76942-76955, (2018).

[8] Liu, J., Sheng, M., Liu, L. and Li, J. Interference management in ultra-dense networks: challenges and approaches. IEEE Network. 31, 6, 70-77 (2017).

[9] Lee, N. and Heath, R. W. Advanced interference management technique: potentials and limitations. IEEE Wireless Communications. 23, 3, 30-38 (2016).

[10] Bohr, N. The quantum postulate and the recent development of atomic theory. Nature, 121, 580-590 (1928).

\section{Acknowledgement:}

The authors would like to thank Prof. Lajos Hanzo, University of Southampton, UK, for fruitful discussions throughout the preparation of this manuscript. 